\title{
Quantum pumping in a ballistic graphene bilayer
}

\author{
G. M. M. Wakker and M. Blaauboer \\ Kavli Institute of Nanoscience, Delft University of Technology, Lorentzweg 1, 2628 CJ Delft, The Netherlands
}

(Received 6 September 2010; published 22 November 2010)

\begin{abstract}
We investigate quantum pumping of massless Dirac fermions in an ideal (impurity free) double layer of graphene. The pumped current is generated by adiabatic variation in two gate voltages in the contact regions to a weakly doped double graphene sheet. At the Dirac point and for a wide bilayer with width $W \gg$ length $L$, we find that the pumped current scales linearly with the interlayer coupling length $l_{\perp}$ for $L / l_{\perp} \ll 1$, is maximal for $L / l_{\perp} \sim 1$, and crosses over to a $\ln \left(L / l_{\perp}\right) /\left(L / l_{\perp}\right)$ dependence for $L / l_{\perp} \gg 1$. We compare our results with the behavior of the conductance in the same system and discuss their experimental feasibility.
\end{abstract}

DOI: $10.1103 /$ PhysRevB.82.205432

PACS number(s): 72.80.Vp, 73.23.Ad

\section{INTRODUCTION}

Quantum pumping of charge refers to the generation of a dc electrical current in the absence of an applied bias voltage by periodic (ac) modulation of two or more system parameters, for example, the shape of the confining potential or a magnetic field. ${ }^{1}$ The idea of adiabatically generating a flow of particles in a moving periodic potential is due to Thouless, ${ }^{2}$ and has been followed by many theoretical and a few experimental investigations of pumping in mesoscopic systems. In 1998 Brouwer, ${ }^{3}$ building on earlier results by Büttiker et al. ${ }^{4}$ and a proposal by Spivak et al. ${ }^{5}$ developed a description of quantum pumping through open mesoscopic systems in terms of the scattering matrix of the system. This paved the way for investigating the effects of quantum interference on quantum pumping and has led to theoretical investigations of many different aspects of pumped currents in open nanodevices, such as the relation of quantum pumping to geometric (Berry) phases, ${ }^{6}$ the effect of Andreev reflection on quantum pumping in nanostructures that contain superconducting parts, $^{7}$ the effect of electron-electron interactions, ${ }^{8}$ and the generation of adiabatically pumped spin currents. ${ }^{9}$

Most of these investigations were carried out for semiconductor structures such as quantum dots and nanowires. In addition, also proposals for adiabatic pumping in carbon nanotubes, ${ }^{10}$ and recently graphene ${ }^{11,12}$ have been put forward. Since its experimental discovery in 2004, ${ }^{13}$ graphene has been found to exhibit electronic transport properties that are quite different from those in other nanoelectronic structures due to the nature of its charge carriers (massless Dirac fermions described by a relativistic wave equation). ${ }^{14} \mathrm{An}$ example of this is the importance of evanescent modes for transport close to the Dirac point: in a sample of undoped graphene, which does not have any free electrons, contacted by doped electrodes the conductance close to the Dirac point is dominated by the contribution of evanescent modes which transmit electrons injected from one end of the sample to the other end. ${ }^{15,16}$ This is also true for quantum pumping in a monolayer of graphene, where the pumped current is induced by two oscillating gate voltages. ${ }^{11}$

In this paper we investigate quantum pumping of Dirac fermions in a graphene bilayer. Compared to a carbon monolayer, the bilayer has an additional energy scale, namely the interlayer coupling strength $t_{\perp}$. The corresponding length scale $l_{\perp}$ is an order of magnitude larger than the interatomic distance $d .{ }^{14}$ Our aim is to investigate the dependence of the adiabatically pumped current on the interlayer coupling $t_{\perp}$. For the conductance in a bilayer with heavily doped contact regions and width $W \gg$ length $L$ it has recently been found that at the Dirac point the bilayer transmits as two monolayers in parallel and the conductance is independent of $t_{\perp} \cdot{ }^{17} \mathrm{In}$ contrast, for the pumped current $I_{p}$ we find that $I_{p}$ depends linearly on $L / l_{\perp}$ for small interlayer coupling strength $t_{\perp}$ $=1 / l_{\perp} \ll 1 / L$, exhibits a maximum around $t_{\perp} \sim 1 / L$ and scales as $\ln \left(L / l_{\perp}\right) /\left(L / l_{\perp}\right)$ for large interlayer coupling $t_{\perp}$ $=1 / l_{\perp} \gg 1 / L$. For typical experimental parameters the pumped current is of order 10-100 pA.

The paper is organized as follows. In Sec. II we present the bilayer model and an introduction to pumped currents. Section III contains the calculation of the pumped current, followed by results and comparison to the conductance in Sec. IV. We conclude by making a connection to experiments in Sec. V.

\section{BILAYER MODEL AND PUMPED CURRENT}

We consider the geometry that is schematically depicted in Fig. 1. A sheet of ballistic graphene in the $(x, y)$ plane contains a weakly doped strip of length $L$ and width $W$ which is contacted by two more heavily doped electrodes at $x=0$ and $x=L$. The doping in these contacts is controlled by gate voltages, which induce a potential profile of the form

$$
U(x)= \begin{cases}U_{1}(t) & \text { for } x<0 \text { or } x>L \text { in the upper layer } \\ U_{2}(t) & \text { for } x<0 \text { or } x>L \text { in the lower layer } \\ 0 & \text { for } 0<x<L \text { in both layers }\end{cases}
$$

We assume the potential step to be abrupt, which is justified close to the Dirac point where the Fermi wavelength $\lambda_{F} \geqslant L$ and any smoothing of the step over a distance small compared to $L$ becomes negligible. In addition, we consider a short and wide geometry $(L \ll W)$, for which boundary conditions in the transverse $y$ direction become irrelevant.

The bilayer pump is operated by periodic variations in the carrier density in the leads by varying the potentials $U_{1}(t)$ $=U_{1}+\delta U_{1} \cos (\omega t)$ and $U_{2}(t)=U_{2}+\delta U_{2} \cos (\omega t+\phi)$ such that 


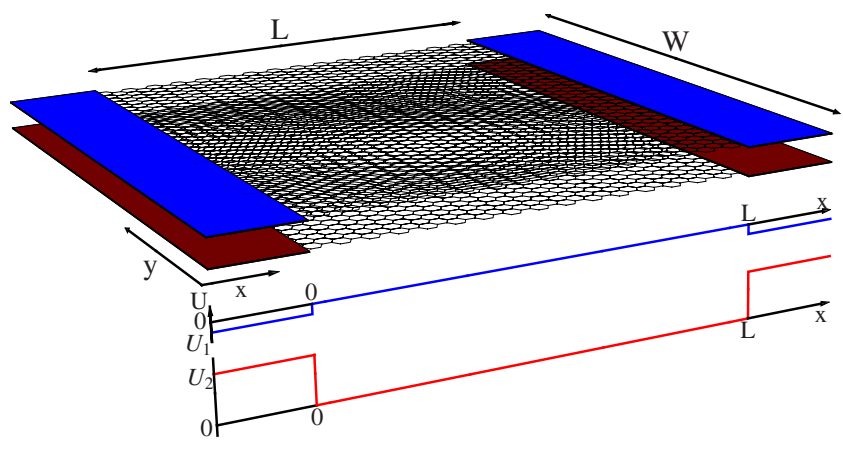

FIG. 1. (Color online) Schematic picture of the graphene bilayer. Top panel: two stacked honeycomb lattices of carbon atoms in a strip of width $W$ between metallic contacts (blue and red regions). Bottom panel: variation in the electrostatic potential across the two layers.

$U_{1}(t)=U_{2}(t)$ on average. In the linear-response regime where $\delta U_{i} \ll U_{i}(i=1,2)$, the pumped current $I_{p}$ into the left lead is given by the scattering matrix expression ${ }^{3}$

$$
I_{p} \equiv I_{L}=\frac{\omega e \sin \phi \delta U_{1} \delta U_{2}}{2 \pi} \sum_{\alpha \in L} \sum_{\beta} \operatorname{Im}\left(\frac{\partial S_{\alpha \beta}^{\star}}{\partial U_{1}} \frac{\partial S_{\alpha \beta}}{\partial U_{2}}\right) .
$$

Here the index $\alpha$ sums over all modes in the left contact region and $\beta$ sums over all modes in both the left and right contact regions. The pumped current into the right lead is then given by $I_{R}=-I_{L}=-I_{p}$. $S$ denotes the Landauer-Büttiker scattering matrix whose elements $S_{\alpha \beta, n m}$ describe scattering from mode $m$ in lead $\beta$ to mode $n$ in lead $\alpha$.

In the presence of a potential $U_{1}\left(U_{2}\right)$ in the upper (lower) layer, the low-energy excitations of the graphene bilayer close to a Dirac point are described by the $4 \times 4$ Hamiltonian,

$$
H=\left(\begin{array}{cccc}
U_{1} & v\left(p_{x}+i p_{y}\right) & t_{\perp} & 0 \\
v\left(p_{x}-i p_{y}\right) & U_{1} & 0 & 0 \\
t_{\perp} & 0 & U_{2} & v\left(p_{x}-i p_{y}\right) \\
0 & 0 & v\left(p_{x}+i p_{y}\right) & U_{2}
\end{array}\right)
$$

where $\mathbf{p}=-i \hbar \partial / \partial \mathbf{r}$ is the momentum operator. Hamiltonian (3) acts on the four-component wave function $\left(\psi_{A_{1}}, \psi_{B_{1}}, \psi_{B_{2}}, \psi_{A_{2}}\right)$, with $A_{1}$ labeling the amplitude on the $A$ sublattice of the first (upper) layer, and similarly for $B_{1}, A_{2}$, and $B_{2}$. We only take nearest-neighbor coupling from $A$ to $B$ sites within the same layer or between the two different layers into account. ${ }^{18}$ The four eigenenergies of Hamiltonian (3) are given by

$$
\begin{aligned}
& \epsilon_{1,2}=\frac{U_{1}+U_{2}}{2}+\frac{1}{2} \sqrt{f_{ \pm}(k),} \\
& \epsilon_{3,4}=\frac{U_{1}+U_{2}}{2}-\frac{1}{2} \sqrt{f_{ \pm}(k)},
\end{aligned}
$$

where

$$
\begin{aligned}
f_{ \pm}(k) \equiv & 4 k^{2}+2 t_{\perp}^{2} \\
& +\left(U_{1}-U_{2}\right)^{2} \pm 2 \sqrt{t_{\perp}^{4}+4 k^{2}\left[t_{\perp}^{2}+\left(U_{1}-U_{2}\right)^{2}\right]}
\end{aligned}
$$

and $k=\left(k_{x}^{2}+k_{y}^{2}\right)^{1 / 2}$ denotes the total momentum. From now onwards, we absorb a factor $(\hbar v)^{-1}$ in $\epsilon, U_{1}, U_{2}$, and $t_{\perp}$, which are all given in units of momentum.

Using scattering matrix theory we calculate in the next two sections the total pumped current ${ }^{19} I_{p}$ for $U_{1}=U_{2} \equiv U$ at the Dirac point $\epsilon=0$ and derive analytic expressions for the limit of heavily doped contacts $U \rightarrow-\infty$ (these were also considered in Ref. 17).

\section{CALCULATIONS}

The scattering matrix $S$ and subsequently its derivatives with respect to $U_{1}$ and $U_{2}$ can be found by matching eigenstates of Hamiltonian (3) at the interfaces $x=0$ and $x=L$. For given $\epsilon$ and transverse momentum $k_{y}$ the eigenstates of Eq. (3) are characterized by two longitudinal momenta $k_{x_{ \pm}}$,

$$
k_{x \pm}=\sqrt{\frac{V_{1}^{2}+V_{2}^{2}}{2} \pm \frac{1}{2} \sqrt{\left(V_{1}^{2}-V_{2}^{2}\right)^{2}+4 t_{\perp}^{2} V_{1} V_{2}}-k_{y}^{2}}
$$

with $V_{j} \equiv \epsilon-U_{j}(j=1,2)$. Associated with each real wave vector $k_{x+}$ are two propagating modes $\phi_{\epsilon,+}^{R}(x, y)$ (right going) and $\phi_{\epsilon,+}^{L}(x, y)$ (left going). Similarly, another two propagating modes $\phi_{\epsilon,-}^{R}(x, y)$ and $\phi_{\epsilon,-}^{L}(x, y)$ correspond to each real wave vector $k_{x_{-}}$. Defining $k_{ \pm} \equiv k_{x_{ \pm}}+i k_{y}$, the left- and rightgoing eigenstates are given by

$$
\begin{gathered}
\phi_{\epsilon, \pm}^{R}(x, y)=N_{ \pm}\left(\begin{array}{c}
X_{2 \pm} V_{1} \\
X_{2 \pm} k_{ \pm}^{*} \\
X_{1 \pm} V_{2} \\
X_{1 \pm} k_{ \pm}
\end{array}\right) e^{i k_{x \pm} x+i k_{y} y}, \\
\phi_{\epsilon, \pm}^{L}(x, y)=N_{ \pm}\left(\begin{array}{c}
X_{2 \pm} V_{1} \\
-X_{2 \pm} k_{ \pm} \\
X_{1 \pm} V_{2} \\
-X_{1 \pm} k_{ \pm}^{*}
\end{array}\right) e^{-i k_{x \pm} x+i k_{y} y},
\end{gathered}
$$

where

$$
\begin{gathered}
X_{j \pm} \equiv V_{j}^{2}-k_{x \pm}^{2}-k_{y}^{2}+t_{\perp} V_{j} \quad j=1,2, \\
N_{ \pm}=\left[2 W k_{x \pm}\left(V_{1} X_{2 \pm}^{2}+V_{2} X_{1 \pm}^{2}\right)\right]^{-1 / 2} .
\end{gathered}
$$

The eigenstates [Eq. (6)] are normalized by $N_{ \pm}$such that each state carries unit current

$$
\frac{I}{e v}=\int_{0}^{W} d y \phi^{\dagger}\left(\begin{array}{cc}
\sigma_{x} & 0 \\
0 & \sigma_{x}
\end{array}\right) \phi \equiv 1 .
$$

For $U_{1}=U_{2} \rightarrow-\infty$, Eqs. (5) and (6) reduce to the results of Ref. 17.

From now onwards we assume to be at the Dirac point $\epsilon=0$. In the undoped graphene region $0<x<L$ we then find from Eq. (5) that $k_{x \pm}= \pm i k_{y}$, which corresponds to evanescent modes. The left-incident eigenstates of Hamiltonian (3) can then be written as 


$$
\psi_{ \pm}(x, y)= \begin{cases}\phi_{\epsilon, \pm}^{R}(x, y)+r_{+ \pm}^{L L} \phi_{\epsilon,+}^{L}(x, y)+r_{- \pm}^{L L} \phi_{\epsilon,-}^{L}(x, y) & \text { for } x<0 \\ {\left[\left(c_{1 \pm} \chi_{1}+c_{2 \pm} \chi_{2}\right) e^{k_{y} x}+\left(c_{3 \pm} \chi_{3}+c_{4 \pm} \chi_{4}\right) e^{-k_{y} x}\right] e^{i k_{y} y}} & \text { for } 0<x<L \\ t_{+ \pm}^{R L} \phi_{\epsilon,+}^{R}(x-L, y)+t_{- \pm}^{R L} \phi_{\epsilon,-}^{R}(x-L, y) & \text { for } x>L .\end{cases}
$$

Here $r_{-+}^{\alpha \beta}$ and $t_{-+}^{\alpha \beta}$ with $\alpha, \beta \in\{L, R\}$ denote the reflection and transmission coefficients from a +-mode incident from lead $\beta$ to a - mode in lead $\alpha$. In the middle region the eigenvectors $\chi_{1}-\chi_{4}$ have been constructed such that they are linearly independent at the Dirac point $\epsilon=0$. This yields

$$
\begin{aligned}
& \chi_{1}=\left(\begin{array}{l}
0 \\
1 \\
0 \\
0
\end{array}\right), \quad \chi_{2}=\left(\begin{array}{c}
0 \\
-i t_{\perp} x \\
1 \\
0
\end{array}\right), \\
& \chi_{3}=\left(\begin{array}{c}
1 \\
0 \\
0 \\
-i t_{\perp} x
\end{array}\right), \quad \chi_{4}=\left(\begin{array}{l}
0 \\
0 \\
0 \\
1
\end{array}\right) .
\end{aligned}
$$

The reflection and transmission coefficients are calculated by matching the eigenstates [Eq. (10)] at the two interfaces $x$ $=0$ and $x=L$, see the Appendix. For a short and wide geometry with $L \ll W$ the boundary conditions in the $y$ direction become irrelevant. Taking infinite mass boundary conditions, such that $k_{y}$ is quantized as $k_{y}=(n+1 / 2) \pi / W, n=0,1,2, \ldots$ the pumped current [Eq. (2)] becomes a sum of eight terms

$$
I_{p}=\frac{\omega e W \sin \phi \delta U_{1} \delta U_{2}}{2 \pi^{2}} \int_{0}^{\infty} d k_{y} \sum_{\sigma, \sigma^{\prime}= \pm} \Pi_{\sigma \sigma^{\prime}}\left(k_{y}\right)
$$

with

$$
\Pi_{\sigma \sigma^{\prime}}\left(k_{y}\right) \equiv \operatorname{Im}\left(\frac{\partial r_{\sigma \sigma^{\prime}}^{* L L}}{\partial V_{1}} \frac{\partial r_{\sigma \sigma^{\prime}}^{L L}}{\partial V_{2}}+\frac{\partial t_{\sigma \sigma^{\prime}}^{* L R}}{\partial V_{1}} \frac{\partial t_{\sigma \sigma^{\prime}}^{L R}}{\partial V_{2}}\right)
$$

\section{RESULTS}

In the Appendix we describe details of the calculation of the derivatives of $r_{\sigma \sigma^{\prime}}^{L L}$ and $t_{\sigma \sigma^{\prime}}^{L R}$ with respect to $U_{1}$ and $U_{2}$ and consider the limit $U_{1} \rightarrow U_{2} \equiv U$ with $U \rightarrow-\infty$. This is equivalent to $V_{1} \rightarrow V_{2} \equiv V$ with $V \rightarrow+\infty$. Substitution of these derivatives into Eq. (12) and integrating over $k_{y}$ then yields the pumped current,

$$
\begin{aligned}
I_{p}= & \frac{\omega e W \sin \phi \delta U_{1} \delta U_{2}}{2 \pi^{2}} \frac{4 \lambda}{U^{2}} \int_{0}^{V \rightarrow \infty} d k_{y} \frac{\left[\lambda^{2}+6 k_{y} L \sinh \left(2 k_{y} L\right)-8 \cosh ^{2}\left(k_{y} L\right)\right] \sinh \left(2 k_{y} L\right)}{\left[\lambda^{2}+4 \cosh ^{2}\left(k_{y} L\right)\right]^{3}} \\
= & \frac{2 \omega e}{\pi^{2}} \frac{W}{L} \lambda \sin \phi \frac{\delta U_{1} \delta U_{2}}{U^{2}} \int_{0}^{V L \rightarrow \infty} d x \frac{\left[\lambda^{2}+6 x \sinh (2 x)-8 \cosh ^{2}(x)\right] \sinh (2 x)}{\left[\lambda^{2}+4 \cosh ^{2}(x)\right]^{3}} \\
= & \frac{\omega e}{2 \pi^{2}} \frac{W}{L} \sin \phi \frac{\delta U_{1} \delta U_{2}}{U^{2}} \frac{1}{4 \lambda^{2}\left(\lambda^{2}+4\right)^{2}}\left\{\lambda^{5}-20 \lambda^{3}+3 \lambda\left(\lambda^{2}+2\right)\left(\lambda^{2}+4\right) \ln \left(\lambda^{2}+4\right)+12 \sqrt{\lambda^{2}+4}\right. \\
& \left.\times\left[L i_{2}\left(2+\frac{\lambda^{2}}{2}+\frac{\lambda}{2} \sqrt{\lambda^{2}+4}\right)-L i_{2}\left(2+\frac{\lambda^{2}}{2}-\frac{\lambda}{2} \sqrt{\lambda^{2}+4}\right)\right]\right\} .
\end{aligned}
$$

Here we have defined the dimensionless coupling length $\lambda \equiv t_{\perp} L$ and $L i_{2}$ is the dilogarithm function defined as $L i_{2}$ $=\int_{1}^{x}(\ln t) /(1-t) d t$. Equation (14c) is the main result of this paper. The upper integration limit in Eq. (14a) originates from the requirement that the wave functions in the left and right leads should correspond to traveling waves, and hence that $k_{x \pm}$ should be real $\left(k_{x-}\right.$ gives the more stringent condition $k_{y} \leq \sqrt{V(V-t)} \approx V$ for $\left.V \rightarrow \infty\right)$. We see that both for $L$ $=0$ and for $L \rightarrow \infty$ the pumped current [Eq. (14c)] reduces to zero. For $L=0$, i.e., in the absence of the middle region, there is no possibility for evanescent waves to interfere in this region: all incoming waves from one lead are fully transmitted into the other lead without scattering to the other layer and hence do not contribute to the pumped current (which is composed of waves that scatter at least once from one layer to the other). For $L \rightarrow \infty$, the evanescent modes in the weakly doped middle layer do not reach the other contact and the pumped current becomes zero.

Figure 2 shows $\Sigma_{\sigma, \sigma^{\prime}= \pm} \Pi_{\sigma \sigma^{\prime}}\left(k_{y}\right)$ [Eq. (A10) in units of $\left.4 \lambda U^{-2}\right]$, which is essentially the mode-dependent pumped 


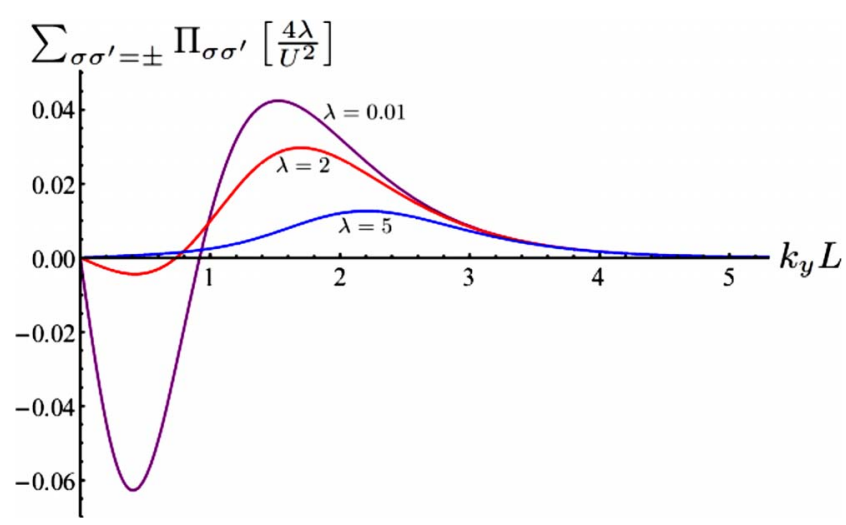

FIG. 2. (Color online) The mode-dependent pumped current [Eq. (A10)] as a function of $k_{y} L$. The curves correspond to $\lambda$ $=0.01$ (purple), $\lambda=2$ (red), and $\lambda=5$ (blue).

current, as a function of $k_{y}$. We see that $\sum_{\sigma, \sigma^{\prime}= \pm} \Pi_{\sigma \sigma^{\prime}}\left(k_{y}\right.$ $=0)=0$, i.e., waves with transverse momentum $k_{y}=0$ that are incident perpendicular to the interface do not contribute to the pumped current, which is a manifestation of the Klein paradox. ${ }^{14,15}$ We also see that negative mode contributions only occur for small values of $\lambda$. From Eq. (A10) it follows that the largest contribution to $\Sigma_{\sigma, \sigma^{\prime}= \pm} \Pi_{\sigma \sigma^{\prime}}$ comes from the transverse modes $k_{y} L \sim 0.1 \lambda$ for $\lambda \sim 50$, similarly as for the transmission (conductance) in the same system. ${ }^{17}$ Figure 3 shows a plot of the total pumped current $I_{p}$ as a function of $\lambda$, for a fixed value of $W / L=100$. For $n \sim 200$ modes the sum converges to the continuum result. The number of modes needed for convergence becomes larger for $W / L$ larger while for $W / L=20$ only $n \sim 20$ modes are needed. As expected, $I_{p}\left(t_{\perp}=0\right)=0$, since in the absence of interlayer coupling the bilayer reduces to two uncoupled monolayers for which the pumping parameters $V_{1}(t)$ and $V_{2}(t)$ become uncoupled and no pumping occurs. From Eq. (14c) we obtain that for small interlayer coupling strength $t_{\perp} \ll 1 / L$ the pumped current $I_{p}$ scales as

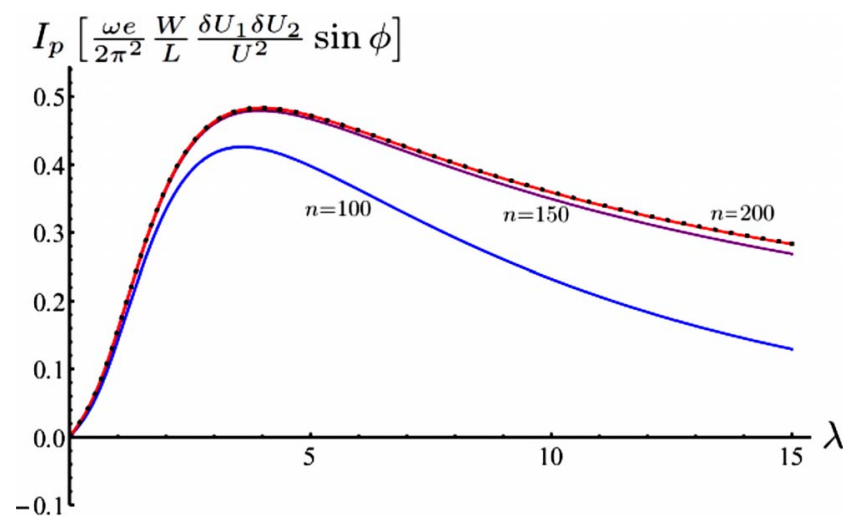

FIG. 3. (Color online) The total pumped current $I_{p}$ as a function of $\lambda$, for $W / L=100$. The blue, purple, and red lines represent the exact sum over $k_{y}$ of the integrand in Eq. (14a) for $n=100,150$, and 200 modes. The black dotted line is the integrated result, Eq. (14c).

$$
\lim _{t_{\perp} \rightarrow 0} I_{p}=\frac{4 \ln 2-1}{64 \pi^{2}} \omega e \frac{W}{L} \sin \phi \frac{\delta U_{1} \delta U_{2}}{U^{2}} \lambda,
$$

hence $I_{p}$ depends linearly on $t_{\perp}$ for $t_{\perp}=1 / l_{\perp} \ll 1 / L$. On the other hand, in the limit of large interlayer coupling $t_{\perp}$ $=1 / l_{\perp} \gg 1 / L$ we find

$$
\lim _{t_{\perp} \rightarrow \infty} I_{p}=\frac{3}{4 \pi^{2}} \omega e \frac{W}{L} \sin \phi \frac{\delta U_{1} \delta U_{2}}{U^{2}} \frac{\ln \lambda}{\lambda},
$$

and hence $I_{p} \sim \frac{\ln t_{\perp} L}{t_{\perp} L}$ in this limit. In between these two limits $I_{p}$ exhibits a maximum which is determined by $d I_{p} / d \lambda=0$ and yields

$$
\lambda_{\max }=3.88 \rightarrow I_{p}\left(\lambda_{\max }\right)=0.51 \frac{\omega e}{2 \pi^{2}} \frac{W}{L} \sin \phi \frac{\delta U_{1} \delta U_{2}}{U^{2}} .
$$

Thus $I_{p}$ is maximal if $t_{\perp}$ is of the same order as $1 / L$. The pumped current thus strongly depends on $t_{\perp}$ and reduces to zero for $t_{\perp} \rightarrow 0$. This is in sharp contrast with the behavior of the conductance at the Dirac point in a graphene bilayer, which equals the conductance across two monolayers and is independent of $t_{\perp} \cdot{ }^{17}$

\section{CONCLUSION}

In summary, we have investigated the adiabatically pumped current through a wide graphene bilayer consisting of a central weakly doped graphene sheet coupled to two heavily doped contact regions. At the Dirac point, the pumped current is carried by evanescent waves in the central region and exhibits a crossover from linear (for $t_{\perp} L \ll 1$ ) to logarithmic (for $t_{\perp} L \gg 1$ ) dependence as a function of increasing interlayer coupling strength $t_{\perp}$, with a maximum around $t_{\perp} L \sim 3.88$. This scaling behavior with $t_{\perp}$ is markedly different from the behavior of the conductance $G$ in the same system, which is independent of $t_{\perp}$ and equal to the conductance across two monolayers in parallel. In practice, this different behavior of $I_{p}$ and $G$ as a function of $t_{\perp}$ and $L$ could be used to distinguish between the conductance and the pumped current.

We can estimate the magnitude of the pumped current using typical experimental parameters ${ }^{14,20,21} t_{\perp}$ $=(0.4 \mathrm{eV}) /\left(\hbar v_{F}\right) \approx 0.6 \times 10^{9} \mathrm{~m}^{-1}, \quad U \approx 0.1 \mathrm{~V}, \quad \delta U \approx 10$ $\mathrm{mV}$, and $\omega \approx 1 \mathrm{GHz}$. In order to be able to measure a substantial pumped current, one thus needs sample sizes $L$ $\sim 4 / t_{\perp} \sim 6 \mathrm{~nm}$, which is smaller than currently available samples of order micron. ${ }^{22}$ However, with steady progress toward smaller sample sizes, sample lengths of order 10-100 $\mathrm{nm}$ are expected to become available in the future. We then obtain from Eq. (14c),

$$
I_{p, \max } \sim 5 \times 10^{-14} \frac{W}{L} \sin (\phi) A,
$$

which, for $W / L \sim 100-1000$ is well within experimental reach. Observation of this current would be a striking demonstration of quantum pumping produced by relativistic quantum mechanics. 


\section{ACKNOWLEDGMENT}

This work has been supported by The Netherlands Organisation for Scientific Research (NWO).

\section{APPENDIX: CALCULATION OF THE SCATTERING MATRIX ELEMENTS AND THEIR DERIVATIVES}

In this appendix we calculate the reflection and transmission coefficients $r_{\sigma \sigma^{\prime}}^{\alpha \beta}$ and $t_{\sigma \sigma^{\prime}}^{\alpha \beta}\left(\sigma, \sigma^{\prime} \in\{+,-\} ; \alpha, \beta \in L, R\right)$ from Eq. (10) and their derivatives with respect to $U_{1}$ and $U_{2}$. These derivatives are then used to calculate the pumped current [Eq. (12)].

Matching the eigenfunctions [Eq. (10)] at the interfaces $x=0$ and $x=L$ results in the eight equations,

$$
\begin{gathered}
\left(\begin{array}{c}
X_{2+} V_{1} \\
-X_{2+} k_{+} \\
X_{1+} V_{2} \\
-X_{1+} k_{+}^{*}
\end{array}\right)+r_{- \pm} N_{-}\left(\begin{array}{c}
X_{2-} V_{1} \\
-X_{2-} k_{-} \\
X_{1-} V_{2} \\
-X_{1-} k_{-}^{*}
\end{array}\right) \\
=\left(\begin{array}{l}
c_{3 \pm} \\
c_{1 \pm} \\
c_{2 \pm} \\
c_{4 \pm}
\end{array}\right)-N_{ \pm}\left(\begin{array}{c}
X_{2 \pm} V_{1} \\
X_{2 \pm} k_{ \pm}^{*} \\
X_{1 \pm} V_{2} \\
X_{1 \pm} k_{ \pm}
\end{array}\right), \quad(\mathrm{A} 1) \\
t_{+ \pm} N_{+}\left(\begin{array}{c}
X_{2-} V_{1} \\
X_{2-} k_{-}^{*} \\
X_{1-} V_{2} \\
X_{2+} k_{+}^{*} \\
X_{1+} V_{2} \\
X_{1+} k_{+}
\end{array}\right)=\left(\begin{array}{c}
c_{- \pm} \\
c_{3 \pm} z^{-1} \\
\left(c_{1 \pm}-i t_{\perp} L c_{2 \pm}\right) z \\
c_{2 \pm} z \\
\left(c_{4 \pm}-i t_{\perp} L c_{3 \pm}\right) z^{-1}
\end{array}\right)
\end{gathered}
$$

with $z \equiv \exp \left(k_{y} L\right), V_{j}=-U_{j}$ at the Dirac point $(j=1,2)$ and $X_{j \pm}$ and $N_{ \pm}$given by Eqs. (7) and (8). Eliminating $c_{1}-c_{4}$ from Eqs. (A1) and (A2) yields after some straightforward but lengthy algebra for the four reflection coefficients,

$$
r_{\sigma \sigma^{\prime}}^{L L}=\frac{\alpha_{\sigma \sigma^{\prime}}^{L L} \cosh \left(2 k_{y} L\right)+\beta_{\sigma \sigma^{\prime}}^{L L} \sinh \left(2 k_{y} L\right)+\gamma_{\sigma \sigma^{\prime}}^{L L}}{\delta \cosh \left(2 k_{y} L\right)+\epsilon \sinh \left(2 k_{y} L\right)+\eta}
$$

with $\sigma, \sigma^{\prime} \in\{+,-\}$. Using $k_{ \pm} \equiv k_{x \pm}+i k_{y}$, the dimensionless coupling length $\lambda \equiv t_{\perp} L, A \equiv X_{1+} X_{2-}$, and $B \equiv X_{1-} X_{2+}$, the coefficients in Eq. (A3) are given by

$$
\begin{aligned}
& \alpha_{++}^{L L}= 2 N_{+} N_{-}\left[i k_{y}\left(k_{+} A^{2}-k_{+}^{*} B^{2}\right)+\left(k_{x+}^{2}-k_{x-}^{2}+2 k_{y}^{2}\right) A B\right], \\
& \beta_{++}^{L L}=2 N_{+} N_{-} k_{x-}\left[k_{+} A^{2}-k_{+}^{*} B^{2}-2 i k_{y} A B\right], \\
& \gamma_{++}^{L L}=N_{+} N_{-}\left[\lambda^{2}(A-B)^{2} V_{1} V_{2}-2 i \lambda(A-B) k_{x-}\left(X_{1+} X_{1-} V_{2}\right.\right. \\
&\left.\left.-X_{2+} X_{2-} V_{1}\right)\right]-\alpha_{++}^{L L}, \\
& \alpha_{+-}^{L L}=2 N_{-}^{2} k_{x-} X_{1-} X_{2-}\left[\left(k_{x+}-k_{x-}+2 i k_{y}\right) A\right. \\
&\left.+\left(k_{x+}-k_{x-}-2 i k_{y}\right) B\right], \\
& \beta_{+-}^{L L}=2 N_{-}^{2} k_{x-} X_{1-} X_{2-}\left(k_{x+}+k_{x-}\right)(A-B),
\end{aligned}
$$

$$
\gamma_{+-}^{L L}=-2 i \lambda N_{-}^{2}(A-B) k_{x-}\left(X_{1-}^{2} V_{2}-X_{2-}^{2} V_{1}\right)-\alpha_{+-}^{L L},
$$

and

$$
\begin{aligned}
& \alpha_{-+}^{L L}=\alpha_{+-}^{L L}(\text { subindex }+\leftrightarrow \text { subindex }-), \\
& \beta_{-+}^{L L}=\beta_{+-}^{L L}(\text { subindex }+\leftrightarrow \text { subindex }-), \\
& \gamma_{-+}^{L L}=\gamma_{+-}^{L L}(\text { subindex }+\leftrightarrow \text { subindex }-), \\
& \alpha_{--}^{L L}=\alpha_{++}^{L L}(\text { subindex }+\leftrightarrow \text { subindex }-), \\
& \beta_{--}^{L L}=\beta_{++}^{L L}(\text { subindex }+\leftrightarrow \text { subindex }-), \\
& \gamma_{--}^{L L}=\gamma_{++}^{L L}(\text { subindex }+\leftrightarrow \text { subindex }-) .
\end{aligned}
$$

Also

$$
\begin{aligned}
& \delta=2 N_{+} N_{-}\left[-\left(k_{x+} k_{x-}-k_{y}^{2}\right)\left(A^{2}+B^{2}\right)+\left(k_{x+}^{2}+k_{x-}^{2}-2 k_{y}^{2}\right) A B\right], \\
& \epsilon=2 i N_{+} N_{-}\left[-\left(k_{x+}+k_{x-}\right) k_{y}\left(A^{2}+B^{2}\right)+2\left(k_{x+}+k_{x-}\right) k_{y} A B\right], \\
& \eta=N_{+} N_{-}\left[-\lambda^{2}(A-B)^{2} V_{1} V_{2}-2 i \lambda\left(k_{x+}-k_{x-}\right)(A-B)\right. \\
& \times\left(X_{1+} X_{1-} V_{2}-X_{2+} X_{2-} V_{1}\right)-2\left(k_{x+} k_{x-}+k_{y}^{2}\right)(A-B)^{2} \\
&\left.-2\left(k_{x+}-k_{x-}\right)^{2} A B\right] .
\end{aligned}
$$

The transmission coefficients in Eq. (A2) are given by

$$
\begin{aligned}
& t_{++}^{R L}=\frac{\mu_{++}^{R L} \cosh \left(k_{y} L\right)-\nu_{++}^{R L} \sinh \left(k_{y} L\right)}{\delta \cosh \left(2 k_{y} L\right)+\epsilon \sinh \left(2 k_{y} L\right)+\eta}, \\
& t_{+-}^{R L}=\frac{\mu_{+-}^{R L} \cosh \left(k_{y} L\right)-\nu_{+-}^{R L} \sinh \left(k_{y} L\right)}{\delta \cosh \left(2 k_{y} L\right)+\epsilon \sinh \left(2 k_{y} L\right)+\eta}, \\
& t_{-+}^{R L}=t_{+-}^{R L}(\text { subindex }+\leftrightarrow \text { subindex }-), \\
& t_{--}^{R L}=t_{++}^{R L}(\text { subindex }+\leftrightarrow \text { subindex -) }
\end{aligned}
$$

with

$$
\begin{aligned}
& \mu_{++}^{R L}=-2 N_{+} N_{-}(A-B) k_{x+}\left[2 k_{x-}(A-B)+i \lambda\left(X_{1+} X_{1-} V_{2}\right.\right. \\
&-\left.\left.X_{2+} X_{2-} V_{1}\right)\right] \\
& \nu_{++}^{R L}=4 i N_{+} N_{-}(A-B)^{2} k_{x+} k_{y} \\
& \mu_{+-}^{R L}=-2 i \lambda N_{-}^{2}(A-B) k_{x-}\left(X_{1-}^{2} V_{2}-X_{2-}^{2} V_{1}\right), \\
& \nu_{+-}^{R L}=2 N_{-}^{2}(A-B) k_{x-} \\
& \times\left[i \lambda\left(X_{1-}^{2} V_{2}+X_{2-}^{2} V_{1}\right)-2 X_{1-} X_{2-}\left(k_{x+}-k_{x-}\right)\right] .
\end{aligned}
$$

In two limiting cases the reflection and transmission coefficients [Eqs. (A3) and (A6a)-(A6d)] reduce to simple forms.

(1) For $L \rightarrow 0$, the + and - modes decouple and we obtain from Eq. (A6a)-(A6d), 


$$
t_{++}^{R L} \rightarrow 1 \quad \text { and } \quad t_{+-}^{R L} \rightarrow 0 .
$$

(2) For $t_{\perp} \rightarrow 0$, i.e., in the absence of interlayer coupling, the reflection and transmission coefficients [Eqs. (A3) and (A6a)-(A6d)] reduce to the monolayer expressions ${ }^{15,16}$

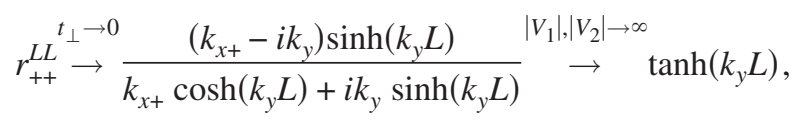

$$
t_{++}^{R L} \stackrel{k_{\perp} \rightarrow 0}{\rightarrow} \frac{k_{x+}}{k_{x+} \cosh \left(k_{y} L\right)+i k_{y} \sinh \left(k_{y} L\right)} \stackrel{\left|V_{1}\right|,\left|V_{2}\right| \rightarrow \infty}{\rightarrow} \frac{1}{\cosh \left(k_{y} L\right)},
$$

$$
t_{+-}^{R L} \stackrel{t_{\perp} \rightarrow 0}{\rightarrow} 0 .
$$

The reflection and transmission coefficients for rightincident Dirac fermions $r_{\sigma \sigma^{\prime}}^{R R}$ and $t_{\sigma \sigma^{\prime}}^{L R}\left(\sigma, \sigma^{\prime} \in\{+,-\}\right)$ are given by Eqs. (A3) and (A6a)-(A6d) by interchanging the layer subindices 1 and 2. So $r_{++}^{R R}=r_{++}^{L L} \quad($ subindex 1 (subindex $1 \leftrightarrow$ subindex 2 ), etc.
In order to calculate the pumped current [Eq. (12)], we need the derivatives of $r_{\sigma \sigma^{\prime}}^{L L}$ and $t_{\sigma \sigma^{\prime}}^{L R}$ with respect to $U_{1}$ and $U_{2}$. These can be calculated by splitting the coefficients $\alpha_{++}^{L L}$, etc., in Eq. (A3) into real and imaginary parts and differentiating each of these with respect to $V_{1}$ and $V_{2}$. The latter does not make any difference for the current since only products of derivatives with respect to $U_{1}$ and $U_{2}$ enter Eq. (12) which are the same as those of the corresponding derivatives with respect to $V_{1}$ and $V_{2}$ (since $V_{j}=-U_{j}$ for $\epsilon=0, j=1,2$ ). The resulting expressions for the derivatives are rather lengthy and therefore not given here. For equal bias voltages $V_{1}=V_{2} \equiv V$ (as required for a true pumping process) and in the limit of $V \rightarrow \infty$, however, an analytic expression for the pumped current $I_{p}$ [Eq. (2) can be derived. This expression is obtained by first taking the limit $V_{1} \rightarrow V_{2}$, for which $X_{1-}$ $\rightarrow X_{2-}=2 t_{\perp} V$ and $X_{1+} \rightarrow-X_{2+}=\left(V_{1}-V_{2}\right)\left(V+\frac{t}{2}\right) \rightarrow 0$ [see Eq. (7)]. We then expand all terms in Eq. (13) in orders of $V_{1}$ $-V_{2}$ (or, equivalently, $X_{1+}$ ) and find that while the numerators contain terms of order $\left(V_{1}-V_{2}\right)^{7}$ and higher, all terms in the denominator are proportional to $\left(V_{1}-V_{2}\right)^{8}$. In the limit of $V$ $\rightarrow \infty$, assuming $V \gg t_{\perp}, k_{y}$ and $V t_{\perp} \gg k_{y}^{2}$, the terms of order $\left(V_{1}-V_{2}\right)^{7}$ in the numerator cancel and we are left with a finite pumped current which to highest order in $V$ amounts to

$$
\sum_{\sigma, \sigma^{\prime}= \pm} \Pi_{\sigma \sigma^{\prime}}\left(k_{y}\right)=\frac{4 \lambda}{U^{2}} \times \frac{\left[\lambda^{2}+6 k_{y} L \sinh \left(2 k_{y} L\right)-8 \cosh ^{2}\left(k_{y} L\right)\right] \sinh \left(2 k_{y} L\right)}{\left[\lambda^{2}+4 \cosh ^{2}\left(k_{y} L\right)\right]^{3}}
$$

Integration of Eq. (A10) over the transverse modes $k_{y}$ then leads to the pumped current [Eq. (14)] in the main text.

${ }^{1}$ B. L. Altshuler and L. I. Glazman, Science 283, 1864 (1999).

${ }^{2}$ D. J. Thouless, Phys. Rev. B 27, 6083 (1983).

${ }^{3}$ P. W. Brouwer, Phys. Rev. B 58, R10135 (1998).

${ }^{4}$ M. Büttiker, H. Thomas, and A. Prêtre, Z. Phys. B: Condens. Matter 94, 133 (1994).

${ }^{5}$ B. Spivak, F. Zhou, and M. T. Beal Monod, Phys. Rev. B 51, 13226 (1995).

${ }^{6}$ J. E. Avron, A. Elgart, G. M. Graf, and L. Sadun, Phys. Rev. B 62, R10618 (2000)

${ }^{7}$ J. Wang, Y. Wei, B. Wang, and H. Guo, Appl. Phys. Lett. 79, 3977 (2001); M. Blaauboer, Phys. Rev. B 65, 235318 (2002); F. Taddei, M. Governale, and R. Fazio, ibid. 70, 052510 (2004).

${ }^{8}$ See, e.g., J. Splettstoesser, M. Governale, J. König, and R. Fazio, Phys. Rev. Lett. 95, 246803 (2005); E. Sela and Y. Oreg, ibid. 96, 166802 (2006).

${ }^{9}$ E. R. Mucciolo, C. Chamon, and C. M. Marcus, Phys. Rev. Lett. 89, 146802 (2002); P. Sharma and P. W. Brouwer, ibid. 91, 166801 (2003); S. K. Watson, R. M. Potok, C. M. Marcus, and V. Umansky, ibid. 91, 258301 (2003).

${ }^{10}$ Y. Wei, J. Wang, H. Guo, and C. Roland, Phys. Rev. B 64, 115321 (2001); M. R. Buitelaar, V. Kashcheyevs, P. J. Leek, V. I. Talyanskii, C. G. Smith, D. Anderson, G. A. C. Jones, J. Wei, and D. H. Cobden, Phys. Rev. Lett. 101, 126803 (2008); V. Zólyomi, L. Oroszlány, and C. J. Lambert, Phys. Status Solidi B 246, 2650 (2009).

${ }^{11}$ E. Prada, P. San-Jose, and H. Schomerus, Phys. Rev. B 80,
245414 (2009); arXiv:1007.3161 (unpublished).

${ }^{12}$ R. Zhu and H. Chen, Appl. Phys. Lett. 95, 122111 (2009).

${ }^{13}$ K. S. Novoselov, A. K. Geim, S. V. Morozov, D. Jiang, Y. Zhang, S. V. Dubonos, I. V. Grigorieva, and A. A. Firsov, Science 306, 666 (2004).

${ }^{14}$ A. H. Castro Neto, F. Guinea, N. M. R. Peres, K. S. Novoselov, and A. K. Geim, Rev. Mod. Phys. 81, 109 (2009).

${ }^{15}$ M. I. Katsnelson, Eur. Phys. J. B 51, 157 (2006).

${ }^{16}$ J. Tworzydło, B. Trauzettel, M. Titov, A. Rycerz, and C. W. J. Beenakker, Phys. Rev. Lett. 96, 246802 (2006).

${ }^{17}$ I. Snyman and C. W. J. Beenakker, Phys. Rev. B 75, 045322 (2007).

${ }^{18}$ Hamiltonians in which also different couplings were taken into account can be found in Ref. 14.

${ }^{19}$ Our pumped current can also be considered as a rectified net dc between the left and right leads that is induced by an ac voltage bias between the top and bottom leads in the geometry of Fig. 1.

${ }^{20}$ E. V. Castro, K. S. Novoselov, S. V. Morozov, N. M. R. Peres, J. M. B. Lopes dos Santos, J. Nilsson, F. Guinea, A. K. Geim, and A. H. Castro Neto, J. Phys.: Condens. Matter 22, 175503 (2010).

${ }^{21}$ M. I. Katsnelson, K. S. Novoselov, and A. K. Geim, Nat. Phys. 2, 620 (2006).

${ }^{22}$ M. F. Craciun, S. Russo, M. Yamamoto, J. B. Oostinga, A. F. Morpurgo, and S. Tarucha, Nat. Nanotechnol. 4, 383 (2009); B. E. Feldman, J. Martin, and A. Yacoby, Nat. Phys. 5, 889 (2009). 